\title{
ASSESSMENT OF NUTRITIONAL AND THERAPEUTIC VALUES IN FLORAL DIVERSITY (FRUITS) AND ITS IMPACT ON HUMAN HEALTH
}

\author{
Ulka Yadav, Shraddha Mittal and Nishchhal Yadav \\ Govt Madhav Vigyan Mahavidyalaya,Ujjain. M.P.,India. \\ Corresponding author Email : ulka.yadav@gmail.com
}

\begin{abstract}
:
What we eat now will affect our health in twenty years time, the time taken for some illnesses such as heart disease and cancer to develop. There are several biologically plausible reasons why consumption of vegetables and fruit might slow or prevent the onset of chronic diseases. Vegetables and fruit are rich sources of a variety of nutrients including vitamins, trace minerals, and dietary fiber, and many other classes of biologically active compounds. These photochemical can have complementary and overlapping mechanisms of action, including modulation of detoxification enzymes, stimulation of the immune system, reduction of platelet aggregation, modulation of cholesterol synthesis and hormone metabolism, reduction of blood pressure, and antioxidant, antibacterial, and antiviral effects. The objective of this paper is to assess the various nutritional and therapeutic values in floral diversity (fruits) and its impact on human health.
\end{abstract}

\section{Keywords:}

Nutritional Value, Therapeutic Values, Floral Diversity, Fruits,Human health

\section{Introduction:}

The human body needs to get a number of different compounds from food, to keep us healthy, fit and active. Fruits provide us with a range of compounds, which help us to protect us from illnesses that take time to develop being involved both with immediate good health and with protection against diseases that develop over a long period of time, such as cancer, heart conditions, stroke and diabetes. Vitamins are essential substances that the human body cannot make for itself so we have to get them from food. Profound deficiency quickly leads to serious illness and lower-than-optimum intake will affect general wellbeing and quality of life. The fat-soluble vitamins $\mathrm{A}, \mathrm{E}$ and $\mathrm{K}$ are stored by the body so although regular intake is needed this does not have to be every day. 
Vitamin A is essential for growth and normal function of the retina of the eye. Red, orange and yellow fruits are a good source of pro-vitamin A, which is converted in the body. Vitamin $\mathrm{K}$, found in fresh green vegetables and fruit, is necessary for normal blood clotting. The water-soluble vitamins $\mathrm{B}$ and $\mathrm{C}$, are not stored in the body so must be eaten frequently to maintain good health. Vitamin B is made up of several compounds essential for a healthy metabolism. Green leafy vegetables, mushrooms and fruit are good sources. The complex comprises B1 (thiamine), B2 (riboflavin), B6 (pyridoxine), B12 (folate) and B3 (niacin). These vitamins have been traditionally grouped together because of similarities in their properties and functions. Vitamin $\mathrm{C}$ is needed for healthy gums, teeth and bones and it helps to speed up wound healing. Acutedeficiency leads to scurvy. Vitamin $\mathrm{C}$, along with vitamin $\mathrm{E}$, is a potent antioxidant; it has a role in enhancing the action of vitamin $\mathrm{E}$ and in aiding the absorption of iron. Good sources of vitamin C are citrus fruits, berries, blackcurrants etc. Minerals are needed in enzyme systems and for other body functions. Potassium, which is mainly derived from fruits and vegetables, has an important role in health. Deficiency leads to muscular weakness, mental confusion and heart problems. Hemoglobin is the substance that makes your red blood cells red and is used to carry oxygen around the body. Meat and fish are good sources of easily absorbed iron. To help improve iron absorption from plant foods it is recommended that you eat vitamin $\mathrm{C}$ rich fruits or drink a glass of fruit juice with the meal.

\section{Material and Method:}

Multi-Nutritional and Therapeutic values provided by Fruits The main goal of fruits consumption is to assure certain nutritive and catalytic needs, whereas other foodstuff groups assure the energetic needs only. The various values provided by fruits consist of: wDietetic value: - It is a natural prophylactic (preventive) or curative quality of certain foodstuffs. It may also be acquired for the dietetic foodstuffs, by applying some technologies and the rigorous 
abidance of the hygienic-sanitary norms, namely some recipes approved by the

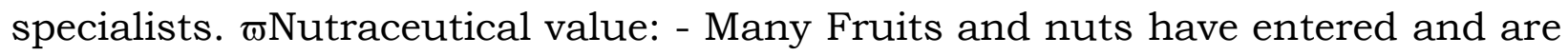
still entering the human alimentation (for instance, nettle, dandelion, etc), as supplements with an auxiliary role in alimentation (vitaminizing, depurative and sometimes, therapeutic one). Initially, their texture or taste were not very pleasant and were not consumed very frequently. Their auxiliary role prevailed over the proper alimentary role, being eaten in order to have a healthy body.

* Medicinal value: - It is subordinated to the Nutraceutical value and certain foodstuff might have medicinal qualities, one of the possible functions of food being also the therapeutic one. There is also an important ethno-botanical tradition of the folk medicine from all the countries that have kept from old times the knowledge about the healing features of fruits and vegetables, picked up from nature or cultivated. Consequences of Not Eating Fruits on Human Health Fruits contribute a host of beneficial nutrients and other food components, such as photochemical and fiber, to your diet. Although you can replace some of them with supplements, no dietary supplement can substitute for all the compounds found in fruits and vegetables, nor can they mimic the potential nutrient interactions found in those foods that may contribute to their healthful effects. Not including these foods as part of a well-balanced diet can result in significant health consequences such as:- *Deficiencies: - One of the initial adverse effects of avoiding fruits might be a vitamin or mineral deficiency. Produce contributes B-vitamins that help you derive energy from your diet, vitamin $\mathrm{C}$ to assist with wound healing, vitamin A to keep your skin and eyes healthy and vitamin K to support blood clotting. Minerals in fruits calcium, magnesium, iron and potassium, which contribute to your skeletal, nerve and cardiovascular health. Avoiding these foods can impact any of these functions. *Digestive Issues: - Fruits contain a type of indigestible carbohydrate called fiber, which doesn't contribute calories to your diet but can improve your intestinal health. Insoluble fiber increases the bulk of waste products in your large intestine, speeds up the waste as it passes through your 
system and helps you avoid constipation and hemorrhoids. Lack of fiber in your diet can have the opposite effect. *Disease Risk: - Soluble fiber swells as it passes through your gut and slows the absorption of nutrients such as glucose and cholesterol. In this way, it can help regulate your blood levels of these molecules and may lessen your risk of diabetes or elevated cholesterol levels. In addition, fruits are rich in photochemical, plant-based substances that not only contribute color to these foods but also may reduce inflammation and even slow or prevent tumor growth. *Cardiovascular Health: - Balancing potassium and sodium in your diet is a key factor in managing your cardiovascular health. Eating plenty of fruits which are rich in potassium, can help restore the balance of these minerals. Not eating these foods may increase your risk of high blood pressure or stroke. Weight Problems: - Fruits have a relatively low energy density, meaning they offer few calories per unit of weight. They therefore provide bulk to your diet but don't overload you with calories, and their water and fiber content helps you feel full and can prevent you from overeating -- particularly helpful if you are trying to lose weight. Excluding fruits in favor of foods with a high energy density, such as cheese or fatty meat, can hinder your efforts to maintain a healthy body weight.

\section{Result and Discussion:}

Benefits of some selected Fruits on human health KIWI- It is a small fruit that protects DNA from mutations. Kiwi an exotic fruit contain as much potassium as bananas. Asthma, wheezing, Coughing and colon cancer especially in children can be prevented. LIME- Lime is a hybrid fruit that cures scurvy, caused due to deficiency of vitamin-C. These are also characterized by infections such as cough and cold, ulcers in mouth, swollen and bleeding gums. The ample of acids present in lime helps to clear the excretory system by cleaning off the tracts LONGAN- Loganberries are high in Vitamin C, Manganese, Vitamin K, Folate, Copper and Dietary Fiber. The fruit is low in saturated fat, cholesterol and sodium. It is used as an ingredient in jams and 
wines. LYCHEE- Lychee fruits are low in calories, contains no saturated fats or cholesterol, but rich in dietary fiber. Canned lychees can be used to make a fruit cocktail. Peeled, seeded lychees are canned in sugar sirup in India and China MANDARIN- Mandarin can be attributed as anti septic, cytophylactic, depurative, digestive, hepatic, relaxant, sedative and tonic. Vitamin $\mathrm{C}$ present in mandarin oranges protects the sperm from genetic damage. Mandarins have been used in ayurveda and to reduce phlegm. MULBERRY- In times past, mulberries were believed to have great curative powers and were used as a general cure-all. Now, they are used mainly in eliminating and weight-loss diets. They are also good for building the blood, and for coating the stomach. NANCE- The fruits are used to prepare carbonated beverages, or an acid, oily, fermented beverage called chicha applied to assorted beer-like drinks. Nance is a tropical fruit cultivated in coastal areas of Mexico. Nance can be used as fresh fruit and for preparing fruit rolls, jellies, syrup, ice cream, and cakes.

OLIVE- The olive oil contains high content of monounsaturated fatty acids and antioxidative substances. People use the fruit, seeds, water of fruit and the leaves to make medicine. It is used to prevent heart attack, stroke, cancer, and migraine headache. ORANGE- Oranges are an excellent source of vitamin C. It is low in calories, no saturated fats or cholesterol, and is rich in dietary fiber, pectin. Orange oil and orange peel extract are key ingredients in some fire ant repellents. Cooking oil is extracted from its seeds. RAISINS- The raisins help in relief from constipation, acidosis, anemia, fever, and sexual weakness. Raisins also help to gain weight, and provides eye care, dental care, and bone health. Raisins may be eaten raw or used in cooking, baking and brewing. RAMBUTAN- Rambutan fruit contains carbohydrate, protein, fat, phosphorus, iron, calcium and vitamin C. The Skin tanin of this fruits contain saponin. The fruit are usually sold fresh, used in making jams and jellies. RASPBERRYRaspberries are considered as the very good cleanser for mucus, for catarrhal conditions, and for toxins present in the body. They are a good source of vitamins $A$ and $C$. Raspberry were called -ida, ll probably after the mountain. 
STRAWBERRY- Strawberries are accessory fruit often used to flavor other foods, such as ice cream. They have many other nutrients and can be efficiently used to prevent many diseases. Strawberry fruit are eaten raw or used in making juice, desserts, jam and syrup. SWEET ORANGE- Sweet Orange is used in alternative herbal treatments to treat ailments and problems. It is used to rejuvenate the skin with the action of the AHA, in skin care cosmetics and to stimulate the lymph system. This orange is used as a rootstock in groves of sweet orange. Cooking oil is extracted from its seeds. WALNUTS- Walnuts are the only nuts that have omega-3 fatty acids in high amounts. Omega -3 fatty acids are helpful in lowering triglycerides. Triglycerides should not be more than a certain level in the blood WATERMELON- Most of us love watermelon for its taste, but many are ignorant of its health benefits. This article deals with the health benefits of watermelon, which is widely cultivated across the globe. Watermelon has been used as a medicinal plant in several native cultures. There are over 50 varieties of watermelon. Analysis of nutritional and therapeutic values in fruits and its impact on human health - Quantitative Assessment of values and its impact Fruit are packed full of goodness and often contain a number of essential vitamins and minerals that cannot be found in other types of foods or they may contain higher levels of these nutrients than other foods. They play a significant role in human nutrition, especially as sources of vitamins [C (ascorbic acid), A, thiamine (B1), niacin (B3), pyridoxine (B6), folacin (also known as folic acid or folate) (B9), E], minerals, and dietary fiber. Their contribution as a group is estimated at $91 \%$ of vitamin C, $48 \%$ of vitamin A, $30 \%$ of folacin, $27 \%$ of vitamin $\mathrm{B} 6,17 \%$ of thiamine, and $15 \%$ of niacin in the diet. Fruits also supply $16 \%$ of magnesium, 19\% of iron, and $9 \%$ of the calories, including riboflavin (B2), zinc, calcium, potassium, and phosphorus which when taken in correct ratio and proportion in the daily diet strongly result in reducing risk for some forms of cancer, heart disease, stroke, and other chronic diseases. Although antioxidant capacity varies greatly among 
fruits it is better to consume a variety of commodities rather than limiting consumption to a few with the highest antioxidant capacity. There is increasing evidence that consumption of whole foods is better than isolated food components such as dietary supplements and nutraceuticals.

\section{Conclusion:}

Fruits are highly nutritious and form as key food commodity in the human consumption. They are highly perishable due to their low shelf but have a natural dietetic value through their significant content in minerals, alimentary fibres, pectic substances, essential amino acids, polyunsaturated fatty acids, vitamins, antioxidants (polyphenols, sulphur compounds, resveratrol), phytoncides (natural antibiotics), etc.At present, more and more nutraceutical foodstuffs are spread (nonalcoholic drinks, spice mixtures, sauces, especially prepared products) that include in their recipes certain components or natural substances with a medicinal effect, namely tonic, stimulating, digestive or protector/antioxidant, extracted from the best known and appreciated cultivated plants or from the spontaneous flora. The medicinal value is subordinated to the nutraceutical value and some foodstuffs may have medicinal qualities, one of the possible functions of a foodstuff being the therapeutic one. There is also an important ethno-botanical tradition of the folk medicine in all the countries, which has preserved from old times the knowledge about the healing features of fruits picked up from nature or cultivate. Thus in nut shell - The beneficial components of fruits reinforce one another and we get best overall effect by eating a wide range of SUPER-HEROS (Fruits) as epidemiologic data and various researches also support the association between high intake of fruits and low risk of chronic disease.ll 


\section{Acknowledgement:}

Thanks to principal, Govt Madhav Vigyan Mahavidyalaya,Ujjain. M.P.,India.

\section{Reference:}

Boileau, T.W., Z. Liao, S. Kim, S. Lemeshow, J.W. Erdman and S.K. Clinton. 2003. Prostate carcinogenesis in N-methyl-N-nitrosourea (NMU)testosterone-treated rats fed tomato powder, lycopene, or energyrestricted diets. J. Natl. Cancer Inst. 95:1578-86.

Craig, W. and L. Beck. 1999. Phytochemicals: health protective effects. Can. J. Diet. Pract. Res. 60:78-84.

Giovannucci, E. 2002. A review of epidemiologic studies of tomatoes, lycopene, and prostate cancer. Exp. Biol. Med. (Maywood) 227:852-9.

Goldberg, G. (ed). 2003. Plants: Diet and Health. The report of a British Nutrition Foundation Task Force. Blackwell Science, Oxford U.K., 347 pp.

Goldman, I.L., A.A. Kader and C. Heintz. 1999. Influence of production, handling, and storage on phytonutrient content of foods. Nutr. Rev. 57:S46-S52.

Gross, J. 1991. Pigments in vegetables: Chlorophylls and carotenoids. AVI

Book, Van Nostrand Reinold Pub. New York NY.

Hyson, D. 2002. The health benefits of fruits and vegetables. A scientific overview for health professionals. Produce for Better Health Foundation, Wilmington DE, 20 pp.

Kalt, W. 2002. Health functional phytochemicals of fruits. Hort. Rev. 27:269315.

Lee, S.K. and A.A. Kader. 2000. Preharvest and postharvest factors influencing vitamin $\mathrm{C}$ content of horticultural crops. Postharv. Biol. Technol. 20:207-220.

Mozafar. A. 1994. Plant vitamins: Agronomic, physiological and nutritional aspects. CRC Press, Boca Raton FL.

Perkins-Veazie, P. and J.K. Collins. 2001. Contribution of nonvolatile phytochemicals to nutrition and flavor. HortTechnology 11:539-546. Prior, R.L. and G. Cao. 2000. Antioxidant phytochemicals in fruits and vegetables; diet and health implications. HortScience 35:588-592. 